\begin{tabular}{|c|l|}
\hline Title & Effect of Magnetic Field of the Precipitation of Ferromagnetic Phase \\
\hline Author(s) & Miyahara, Syôhei; Mitui, Taday asu \\
\hline Citation & 北海道大學理學部紀要, 4(4), 275-286 \\
\hline Issue Date & 1953-11 \\
\hline Doc URL & http://hdl.handle.net/2115/34214 \\
\hline Type & bulletin (article) \\
\hline File Information & 4_P275-286.pdf \\
\hline
\end{tabular}

Instructions for use 


\title{
Effect of Magnetic Field of the Precipitation of Ferromagnetic Phase
}

\author{
Syôhei MIYAHARA and Tadayasu MITUI \\ (Received Aug. 19, 1953) \\ Department of Physics, Faculty of Science, \\ Hokkaido University
}

\begin{abstract}
In this paper, the behaviour of ferromagnetic precipitates in non-ferromagnetic phase and the mechanism of the heat treatment in the magnetic field, i. e. so-called field cooling were studied. For the above purpose, we used the alloy of copper including ca. $2 \%$ of cobalt as the specimen and obtained the interesting results by the torsion method. The maximum magnetic anisotropy of precipitates of ferromagnetic phase is observed, when the field bas been applied between $765^{\circ} \mathrm{C}$ and $715^{\circ} \mathrm{C}$. At this temperature region the dependency of the magnetic anisotropy to the strength of the applied magnetic field have a tendency of saturation in the high magnetic field. For the effect of tempering time within magnetic field at $750^{\circ} \mathrm{C}$, the magnetic anisotropy increases until it reaches the maximum value by the tempering of ca. 5 hours, after that it decreases exponentially to zero througn more tempering times and on the other hand the magnetic anisotropy decreases exponentially by tempering without magnetic field at the same temperature, as is shown in Fig. 5 .
\end{abstract}

\section{\$1. Introduction}

It has been shown by NEx, that the precipitate of which intrinsic magnetization differs from the matrix must possess such property that the sum of the boundary and the magnetostatic energies would be minimum, so that the alloy shows the magnetic anisotropy when the precipitation takes place under magnetic field. Especially the case, that the non-ferromagnetic phase precipitates in the ferromagnetic phase, is of importance to explain the magnetic anisotropy of Alnico $V$ heat-treated under a magnetic field. Recently, detailed studies of the physical properties including the magnetic anisotropy of Alnico $V$ have been made by R. D. HeIdenREICH and E. A. NesirTT ${ }^{23)}$ for the purpose to examine experimentally a current theory of precipitation which accounts for the unusual permanent magnet properties of the alloy. Their experiments have 
clarified various interesting phenomena in precipitations, however, the specimen they used, Alnico V, has not yet been metallographically thoroughly investigated and we have little information as to the precipitate phase of the alloy.

Now, it is interesting to test, if NéEI's consideration would also hold in the inversed case i. e., the ferromagnetic phase precipitates in the non-ferromagnetic matrix. From this point of view we triad to clarify experimentally the behaviour of ferromagnetic precipitates and mechanism of the heat treatment in the magnetic field, i.e., so-called "field cooling".

For the above mentioned purpose the alloy may be metallographically simple and the precipitate must have a high Curie point, then we take the alloy of copper including ca. $2 \%$ of cobalt as the most appropriate substance.

\section{\$2. Preparation of Specimens and Experimental Apparatus}

The alloy used was prepared by melting electrolytic copper with $2 \%$ of pure cobalt by TAMMANN's furnace in a hydrogen atmosphere, then it was pulled out and cooled in the air to the room temperature. The actual composition of the alloy is by chemical analysis: $97.69 \% \mathrm{Cu}$ and $2.31 \% \mathrm{Co}$. Then, the ingot was forged to avoid segregation and internal cavity. Plates were cut from the forged ingot so that their planes were parallel to the hammered plane and they were cut into disks of $13.3 \mathrm{~mm}$ in diameters and $6.4 \mathrm{~mm}$ in thicknesses by a lathe. Then, to eliminate the internal strain due to forging, they were annealed at $1000^{\circ} \mathrm{C}$ for 15 hours in vaccum. Absence of internal cavities or any other imperfections was secured by X-ray transmission photograph. The apparent magnetic anisotropy caused from the irregular shape of the specimen was preliminarily examined by the same torsion method as described later. The specimen prepared by the above mentioned procedure, showed ferromagnetic properties after the slow cooling from $1000^{\circ} \mathrm{C}$. On the other hand, the one showed non-ferromagnetic properties when it was quenched from $1000^{\circ} \mathrm{C}$ in cold water. This fact coincides satisfactorily with the result obtained by G. TAMmann and W. OEISEN.) The ferromagnetic specimens thus obtained showed no anisotropy, this fact confirms that the irregularity in the shape of 
the specimen is negligible.

For the measurement of anisotropy the torsion method was used. That is to say, a disk-form specimen is placed in a strong and uniform magnetic field and the torques necessary to fix the disk arbitrary directions are measured. The measuring apparatus is illustrated in Fig. 1.

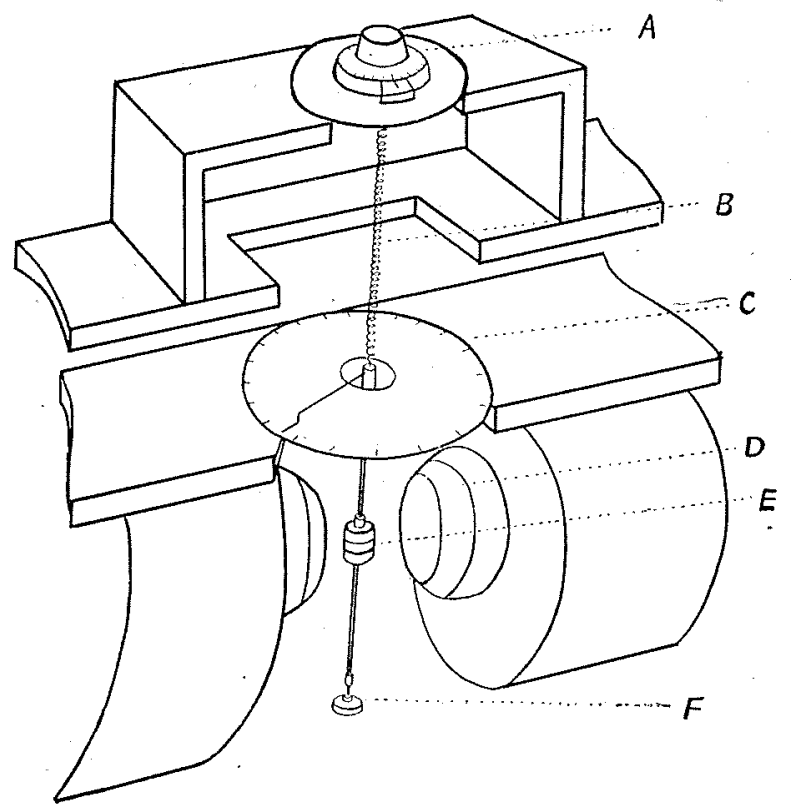

Fig. 1. Measuring Apparatus
A: Dial 1
$D$ : Pole piece of Electromagnet
$B$ : Phosphorbronze wire
$E$ : Specimen
$C$ : Dial 2
$F$ : Jewel Bearing

There, $A$ is a specimen holder which is free to rotate about a vertical axis, and suspended by a helical spring $B$ made of a phosphor bronze wire to whose lower end a pointer is attached so that the angular position of the specimen referred to the direction of magnetic field may be read on a scale of the dial $2 . F$ is a jewel bearing fixed on a solid framework. The upper end of the spring is fixed to the dial 1. The angular twist of the suspension spring can be measured by means of the relative displacement of the scales of dial 1 and dial 2, as the torque acting on the specimen can be 
determined for any desired direction of the specimen. The magnetic field is prepared by an electromagnet of WeIss's type. The specimen is placed in the specimen holder so that the plane of disk is normal to the axis of rotation.

From the measurement of twisted angle of spring, the torque per unit volume is computed as follows:

$$
L=C \Theta_{m}=T(\theta, H) \cdot V_{S}
$$

where $L$ is the torque, $C$ is the torsional constant of the spring, $\Theta_{m}$ is the differences of scales in the dial 1 and dial $2, T(\theta, H)$ is torque per unit volume of the specimen, $\theta$ is the angle between the applied field and the direction of easy magnetization, which might be coincide with the direction of measuring magnetic field as the field is strong enough, and $V_{S}$ is the volume of the specimen. The torsional constant $C$ of the spring was given from the similar torque measurement by a nickel needle replaced with the specimen. From the given values of $C$ and the expression (1), torque per unit volume or anisotropy energy of the specimen will be known.

\section{§3. Experimental Results}

\section{(1) The temperature effective to the precipitation. ${ }^{5}$ )}

The specimens were annealed in non-inductive electric furnace at $1000^{\circ} \mathrm{C}$ for an hour in hydrogen atomsphere and cooled down by the rate of $5^{\circ} \mathrm{C}$ per minute to $200^{\circ} \mathrm{C}$ out of or under the magnetic field of ca. 10,000 oersteds between pole pieces of an electromagnet, taking care that the field direction lies on the disk plane, then the magnetic anisotropy of the specimens was measured. The specimen cooled by the above mentioned rate out of the magnetic field showed no measurable anisotropy. But the one cooled by the same rate under the magnetic field showed remarkable magnetic anisotropy, though the polycrystalline disk should not usually have any definite directions which predominate magnetically. The torque curves of this case are shown in Fig. 2.

Thus we could find the general aspect of the effect of magnetic field for the precipitation, then we tried to find the temperature region effective to the magnetic treatment. Thus, the field was 
applied to the specimen not for whole temperature region but limitedly for each of the consecutive temperature regions devided by $50^{\circ} \mathrm{C}$ from $900^{\circ} \mathrm{C}$ to $200^{\circ} \mathrm{C}$; and not only the case, that the specimen was cooled in the same rate after the field was removed also the case that it was quenched into the cold water when the field was removed off, were together studied. Unexpectedly no measurable effect was observed by the quenching. This fact can not be understand, unless one suppose the heat treatment in the lower temperatures hardly effects the size or shape of precipitates. The results are also shown in Fig. 2.

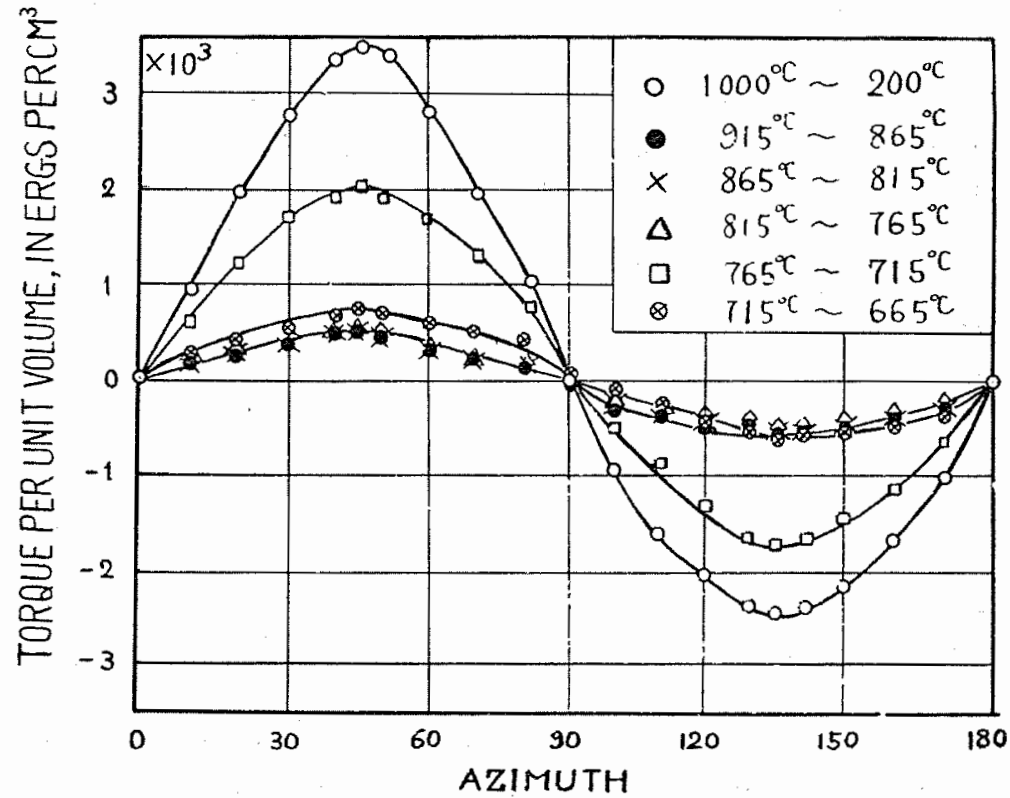

Fig. 2. Torque curves for each of the consecutive temperature regions, in the magnetic field of ca. 10,000 oersteds, measured at room temperature in the magnetic field of 10,000 oersteds.

The greatest anisotropy is observed, when the field has been applied between $765^{\circ} \mathrm{C}$ and $715^{\circ} \mathrm{C}$, as is shown in Fig. 2, where the torque curves of the specimen treated in the field in different temperature regions are together shown. The azimuth is measured from the direction of the field during cooling. Thus, we could find the temperature most effective to the precipitation, and the following experiments were made limitedly at that temperature. 
(2) The effect of strength of the magnetic field.

At the temperature region between $765^{\circ} \mathrm{C}$ and $715^{\circ} \mathrm{C}$, the dependency of the magnetic anisotropy to the strength of the applied magnetic field was measured. Other conditions are same to the former experiments. The result obtained is shown in Fig. 3. In the high field region the increase of anisotropy is relaxed showing a tendency of saturation.

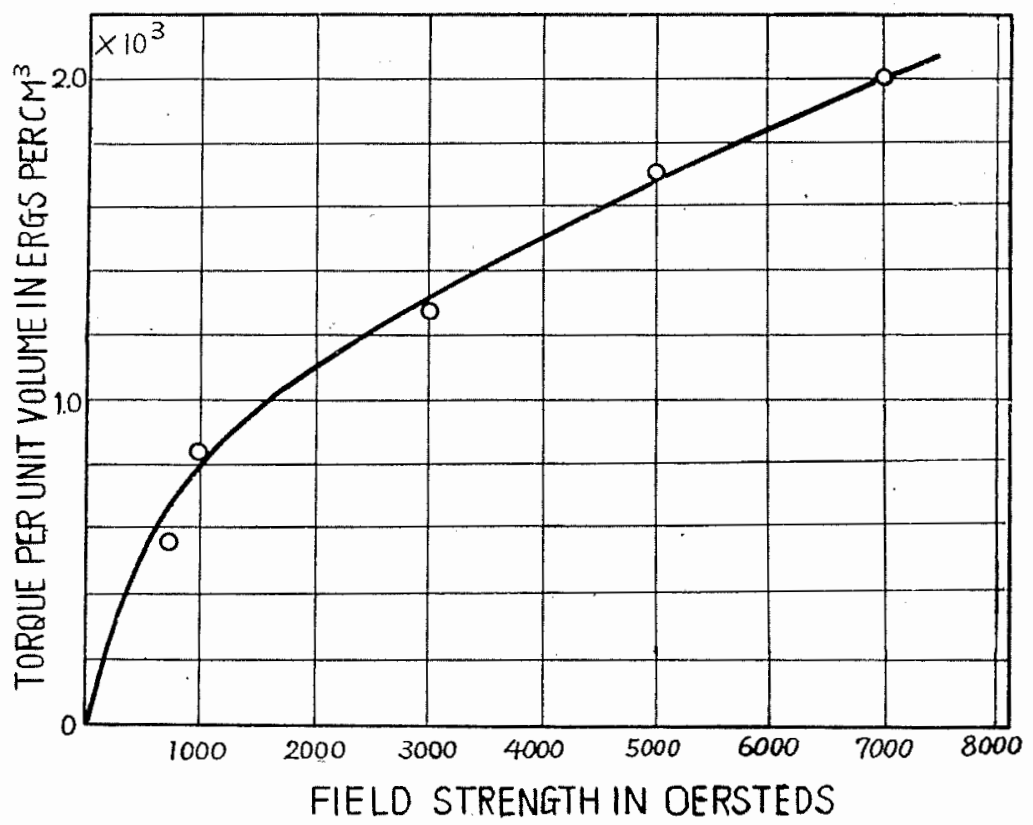

Fig. 3. Variation of magnetic anisotropy with various strengths of the applied magnetic field cooling at the temperature region between $765^{\circ} \mathrm{C}$ and $715^{\circ} \mathrm{C}$, measured at room temperature in the magnetic field of 10,000 oersteds.

\section{(3) Effect of the tempering time.}

In order to clarify the mechanism of precipitation of ferromagnetic phase under magnetic field, we observed the relation of the magnetic anisotropy to the time intervals of isothermal tempering at $750^{\circ} \mathrm{C}$ under the constant magnetic field of $1000 \mathrm{oer}-$ steds. Results obtained are illustrated in Fig. 4. 
The magnetic anisotropy. increases abruptly until it reaches the maximum value with the tempering time of ca. 5 hours, then it decreases exponentially to zero.

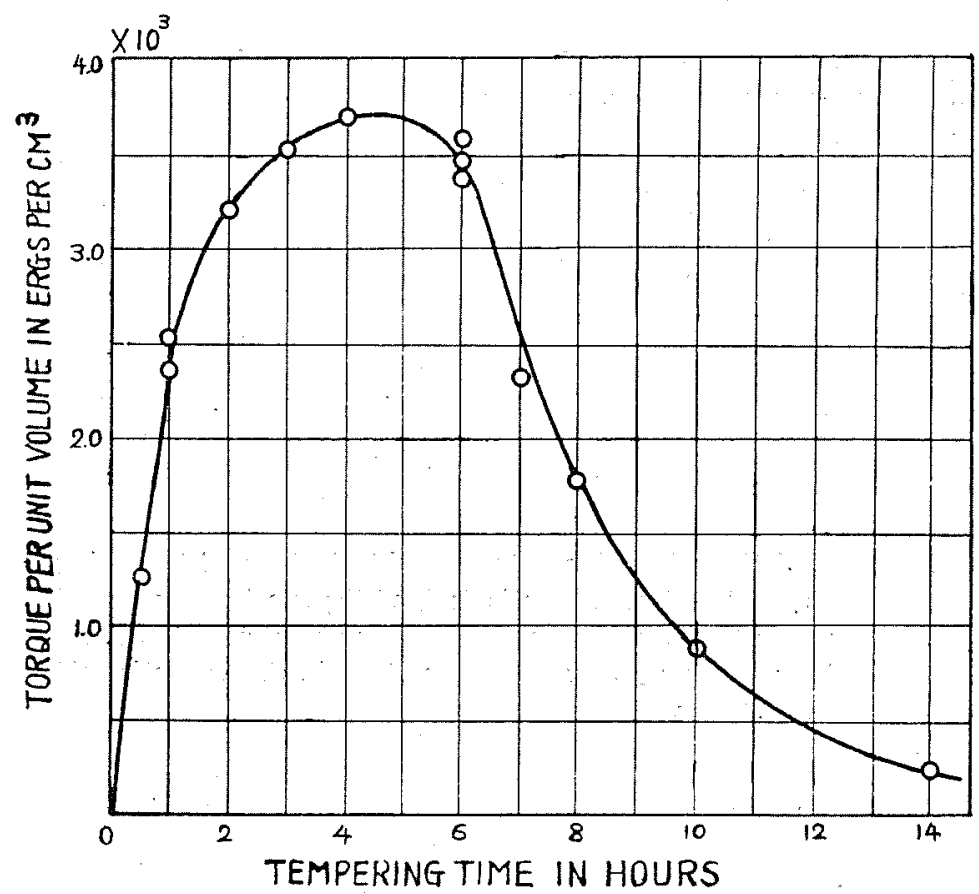

Fig. 4. Variation of maximum torque with the time of tempering at $750^{\circ} \mathrm{C}$ under the constant magnetic field, 1000 oersteds, measured at room temperature in the magnetic field of 10,000 oersteds.

If the magnetic field is get off on the way of the tempering, the aspect of the curve quite differs, e.g. the specimen which was tempered at $750^{\circ} \mathrm{C}$ for 1 hour and showed the maximum torque value $2.1 \times 10^{3} \mathrm{ergs} / \mathrm{cm}$, is followed by tempering without field at the same temperature; then the maximum torque decreases exponentially, as is shown in Fig. 5. 


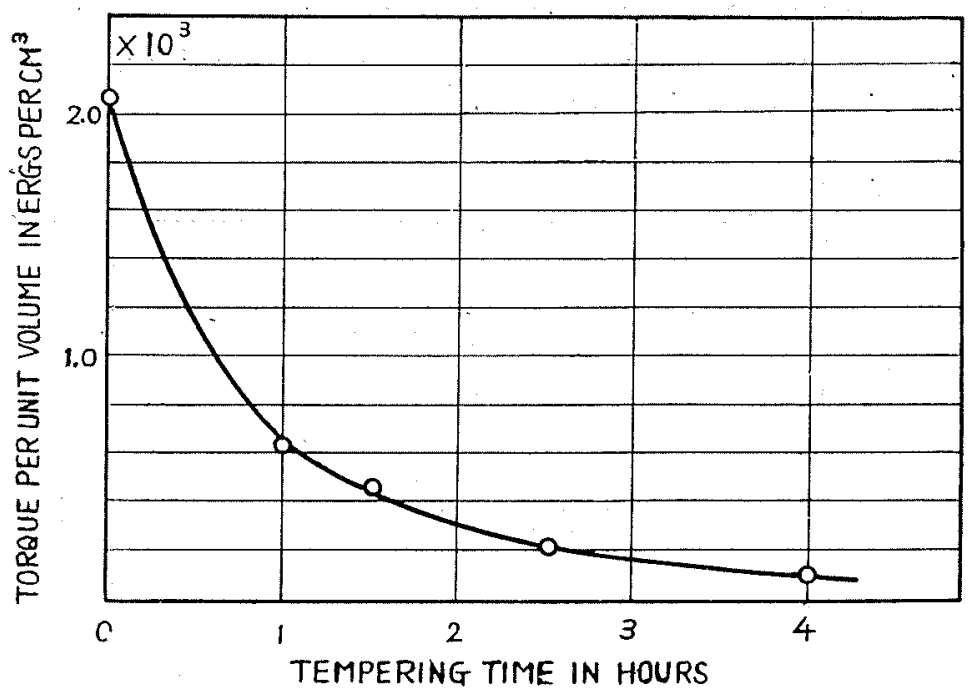

Fig. 5. Variation of maximum torque with the time of tempering at $750^{\circ} \mathrm{C}$ without magnetic field, of the specimen tempered under the magnetic field of 1000 oersteds at $750^{\circ} \mathrm{C}$ for 1 hour, measured at room temperature in the magnetic field of 10,000 oersteds.

\section{§4. Microscopic Examination}

The light microscopic photographs were taken by the specimen electrolytically polished and etched, in order to observe directly the behaviour of precipitates of ferromagnetic phase in non-ferromagnetic phase. The procedure adopted as a standard preparation for light microscopic metallography of the specimen is the method originated by $\mathrm{J}_{\mathrm{ACQUET}}$ and used on copper by LowERY, WiLKINSON and SMARE. ${ }^{6)}$ The electrolyte used in this method was orthophosphoric acid with a specific gravity of 13.5 and the polishing time varied from 5 to 10 minutes and the etching took about 2 minutes at 1.2 volt and $6 \sim 8 \mathrm{~A} / \mathrm{dm}^{2}$.

In Fig. 6 the light microscopic photograph shows acicular type of precipitates of ferromagnetic phase obtained by slow cooling from $1000^{\circ} \mathrm{C}$ to room temperature with magnetic field. Planes of precipitates confirmed from this micrograph and these are (111)planes. 


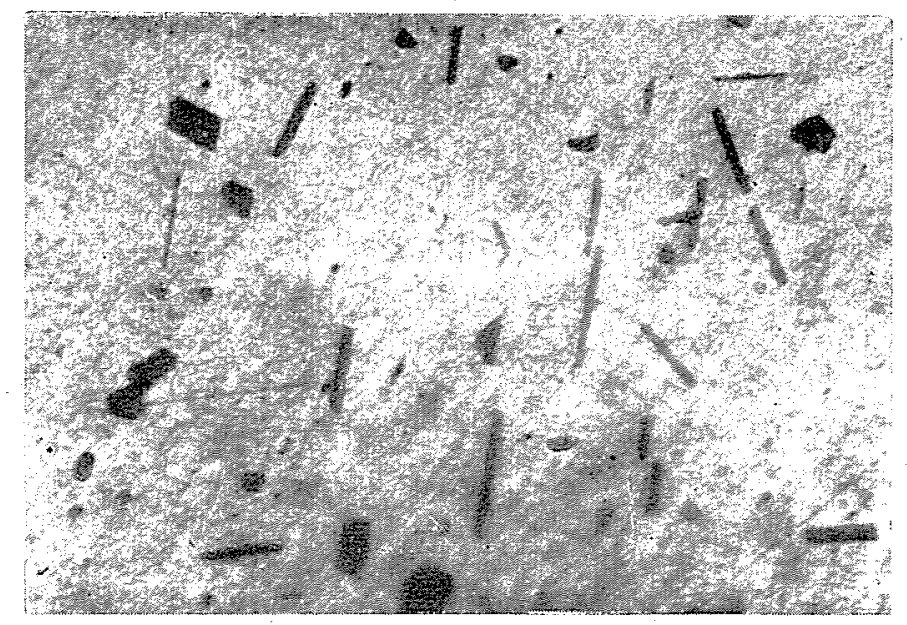

Fig. 6. Light micrograph $(\times 530)$ of slowly magnetic cooled $\mathrm{Cu}-\mathrm{Co}$ alloy showing acicular type of precipitates of ferromagnetic phase.

\section{Discussions}

The experiments above stated have clarified, that the magnetic field have influence upon the precipitation of the ferromagnetic phase, so that the specimens show the uniaxial anisotropy. This effect is similar to those which are observed by HeIdenreich and Nesuits in Alnico V.

The mechanism of the induced anisotropy of our alloy might be explained by NkEr's theory as well as the case of Alnico V. The detailed examination of our experiments, however, forces us to improve the theory.

In the first place, as stated in $\$ 3,(3)$, the anisotropy inclined to disappear for the prolonged tempering. If the anisotropy were caused by the shape and the orientation of the precipitates to minimized the sum of the magnetostatic and the boundary energies, it should continuously increase untill it reaches the definite value during the tempering. In our case, however, the field is likely to be effective only in the beginning of the precipitation process.

Secondly, the shape or orientation of the precipitates does not intimately related to the affected field, as mieroscopic examination shows, but related to the crystal axis of the matrix. 
Then we suppose that the boundary energy of the precipitates will be sensitively dependent on the orientation of their surface in the matrix, so that the shape and the orientation of the precipitates are nearly decided by the crystal axis of the matrix. Only in the initial stage of the precipitation, that is to say, the nucleation of precipitation, in which the volume of the precipitates is so small that the magnetostatic and the thermal energies are comparable in order, the magnetostatic part of the free energy plays the role to bring up favorable nuclei and confines unfavorable ones. Then we are likely to speak, that the magnetic field can only make a selection of the favorable precipitates.

If the precipitates be acicular (or lamellar), we can assume that they have greatest (or least) susceptibility only to one direction. (This assumption is equivalent to the assumption of the fundamental uniaxial anisotropy). Then the magnetization of a disk of the material will be

$$
I=H \sum_{i} \chi_{i} \cos \phi_{i}
$$

where $z_{i}$ means the average susceptibility of acicular precipitates in certain direction $i$, and $\psi_{i}$ is the angle between the acicular axis and the measuring field. Or the parallel and perpendicular components of the magnetization to the direction of the measuring field are respectively

$$
I_{11}=H \sum_{i} \chi_{i} \cos ^{2} \phi_{i}
$$

and

$$
I_{L}=H \sum_{i} \chi_{i} \cos \psi_{i} \sin \psi_{i} .
$$

If we assume the susceptibility $\chi_{i}$ depends only upon the angle $\theta_{i}$ between the acicular axis $i$ and the affected magnetic field during tempering,

$$
I_{11}=H \sum_{i} \chi_{i}\left(\theta_{i}\right) \cos ^{2} \psi_{i}
$$

and

$$
I_{\perp}=H \sum_{i} \chi_{i}\left(\theta_{i}\right) \cos \phi_{i} \sin \phi_{i} .
$$

The susceptibility $\chi_{r i}$ may naturally take the form $\chi\left(\theta_{i}\right)=\chi\left(\left|\cos \theta_{i}\right|\right)$, and moreover we assume 


$$
\chi\left(\theta_{i}\right)=\chi_{0}\left|\cos \theta_{i}\right|,
$$

(that is equivalent that the susceptibility of direction $i$ is proportional to the component of the affected magnetic field in the direction $i$ ), then

$$
I_{11}=H \sum_{i} \chi_{0}\left|\cos \theta_{i}\right| \cos ^{2} \psi_{i},
$$

and

$$
I_{\perp}=H \sum_{i} \chi_{0}\left|\cos \theta_{i}\right| \cos \psi_{i} \sin \psi_{i} .
$$

If we use the polycrystalline specimen, $\theta_{i}$ and $\phi_{i}$ may distribute equally to all directions. Then

$$
\begin{aligned}
& I_{1 \mid}=H \chi_{0} \int_{0}^{2 \pi} d \varphi \int_{0}^{\pi}|\cos \theta| \cos ^{2} \psi \sin \theta d \theta \\
& I_{\perp}=H \chi_{0} \int_{0}^{2 \pi} d \varphi \int_{0}^{\pi}|\cos \theta| \cos \psi \sin \psi \sin \theta d \theta
\end{aligned}
$$

with

$$
\cos \psi=\cos \theta \cos \alpha-\sin \alpha \sin \theta \cos \varphi
$$

where $\alpha$ is the angle between affected field and measuring field, and $\varphi$ is the angle between the direction $i$ and the normal of the plane of the disk. It is easily shown in this case, $I_{\| 1}$ takes the form of angular dependency such as $1+\cos ^{2} \alpha$, and $I_{\perp}=K \cos \alpha \sin$ $\alpha$. In the case of a single crystal, the equation (2) shows greater anisotropy than the case of polycrystals.

On the other hand if the field would not affect the precipitation, i.e. the correlation force between the precipitate and the matrix is exceedingly large, then we can obtain no anisotropy.

There may be the different case, in which the correlation of the precipitates and the matrix is small, so that the magnetic field is able to rotate the direction of the acicular precipitates off from the crystalographic axis of the matrix, as Heidenreich and Nesistr have found in Alnico V. Extremely, when the matrix has no directional effect, Nír,'s original consideration will be left perfectly correct and there should be no difference between single and polycrystals. 


\section{§. Acknowledgement}

In the beginning of this work, we are much indebted to $\mathrm{Mr}$. A. SugiYaMA and Mr. Y. SAWAguti, to whom our hearty thanks are expressed. We thank also Prof. S. KODA and Assist. Prof. R. WADA of the Department of Metallurgy for the helps and advices in metallographical matters, and to $\mathrm{Mr}$. M. Asanuma and Mr. Y. HamaGUTI for the preparation of the specimens, and to Mr. N. Sakamoto for fhe preparation of the measuring apparatus throughout the course of this work.

The part of the expence of the research is defrayed from the Special Fund for the Scientific Research of the Educational Ministry of Japan.

\section{References}

1) L. Néxs, Comp. rend. 225 (1947).

2) R. D. Hidnenkeicn and E. A. Nesmire, J. Appl. Phys. 23 (1952), 352.

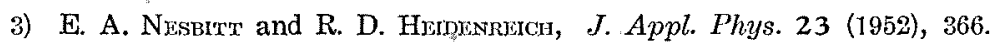

4) G. Tammanin and W. Omlissin, Z. Anorg. Chem. 186 (1930), 257.

5) S. Mryanara and T. Mruu, J. Phys. Soc. Japan 7 (1952), 534.

6) H. Lowery, H. Willinson and D. L. Smare, Phil. Mag. 22 (1936), 769. 